BMJ Open

Diabetes

Research

\& Care

\title{
Diabetes mellitus among adults with tuberculosis in the USA, 2010-2017
}

\author{
Lori R Armstrong, J Steve Kammerer, Maryam B Haddad
}

To cite: Armstrong LR, Kammerer JS, Haddad MB. Diabetes mellitus among adults with tuberculosis in the USA, 2010-2017. BMJ Open Diab Res Care 2020;8:e001275. doi:10.1136/ bmjdrc-2020-001275

Received 11 February 2020 Revised 16 May 2020 Accepted 2 June 2020

\section{Check for updates}

(C) Author(s) (or their employer(s)) 2020. Re-use permitted under CC BY-NC. No commercial re-use. See rights and permissions. Published by BMJ.

Division of Tuberculosis Elimination, Centers for Disease Control and Prevention, Atlanta, Georgia, USA

Correspondence to Dr Maryam B Haddad; mhaddad@cdc.gov

\section{ABSTRACT}

Introduction To describe diabetes trends among adults with incident tuberculosis (TB) disease and examine diabetes-associated TB characteristics and patient outcomes in the USA.

Research design and methods We examined all 71855 persons aged $\geq 20$ years with incident TB disease reported to the National Tuberculosis Surveillance System during 2010-2017. We performed multivariable logistic regression, comparing characteristics and outcomes among patients with TB reported to have diabetes and those whose diabetes status was unknown.

Results An overall $18 \%(n=13281)$ of the 71855 adults with incident TB disease were reported as also having diabetes; the annual proportion increased from $15 \%$ in 2010 to $22 \%$ in 2017 . Among patients aged $\geq 45$ years with both TB and diabetes, the adjusted OR for cavitary or sputum smear-positive TB was 1.7 and 1.5, respectively (95\% Cls 1.5 to 1.8 and 1.4 to 1.6). Patients with TB and diabetes had $30 \%$ greater odds of dying and took longer to achieve negative Mycobacterium tuberculosis cultures and complete treatment.

Conclusions The prevalence of reported diabetes among adults with TB disease has increased. Having diabetes as a comorbidity negatively affects patient outcomes. In accordance with national recommendations, all patients aged $\geq 45$ years and all younger patients who have risk factors for diabetes should be screened for diabetes at the start of TB treatment.

\section{INTRODUCTION}

For persons infected with Mycobacterium tuberculosis, having diabetes is thought to triple the risk of developing tuberculosis (TB) disease. ${ }^{1}$ Worldwide growth in diabetes prevalence is counteracting many countries' otherwise successful TB control strategies. ${ }^{1}$ More advanced, infectious forms of TB disease is associated with lung cavities that are visible on chest radiograph and sputum that is smear positive for acid-fast bacilli. ${ }^{2}$ An international meta-analysis of pulmonary TB's radiologic presentations found that compared with patients without diabetes, those with diabetes had more cavitary lesions. ${ }^{3}$ Prospective studies of newly diagnosed $\mathrm{TB}$ cases in Mexico and China found that compared with patients with TB without diabetes, those with diabetes who had started TB treatment experienced delays in achieving negative sputum

\section{Significance of this study}

What is already known about this subject?

- This is the first national analysis describing diabetes among persons with incident tuberculosis (TB) disease in the USA.

What are the new findings?

- During 2010-2017, 18\% of adult patients with TB in the USA were reported as also having a diabetes diagnosis.

- Non-US-born patients with TB aged $\geq 45$ years were reported as having the highest prevalence of diabetes.

- Consistent with studies in other parts of the world, patients with TB who also had diabetes had more severe TB presentations $(70 \%$ greater odds of having cavitary TB and $50 \%$ greater odds of smearpositive TB).

- Patients with TB and diabetes had greater odds of death; among adults who did not die, however, TB treatment completion was $>93 \%$.

How might these results change the focus of research or clinical practice?

- These findings underscore the national recommendation for baseline diabetes screening among all patients with TB aged $\geq 45$ years and all younger patients who have risk factors for diabetes.

smears and cultures for M. tuberculosis. They were also more likely to die. ${ }^{45}$ This greater risk for death was also the conclusion of an international systematic review and a recent analysis of TB cases in California. ${ }^{67}$

Since 1953, the Centers for Disease Control and Prevention has worked with local and state health departments to collect information about each case of TB disease in the USA. TB incidence has declined since $1993 .^{89}$ However, because of increased concern about diabetes' role as a frequent TB comorbidity, the National Tuberculosis Surveillance System (NTSS) added diabetes status to the standardized TB case data collection form in 2009. ${ }^{9}$ This is the first national analysis since the addition of that diabetes variable. We describe diabetes trends among adults with incident TB disease during 2010-2017 and 


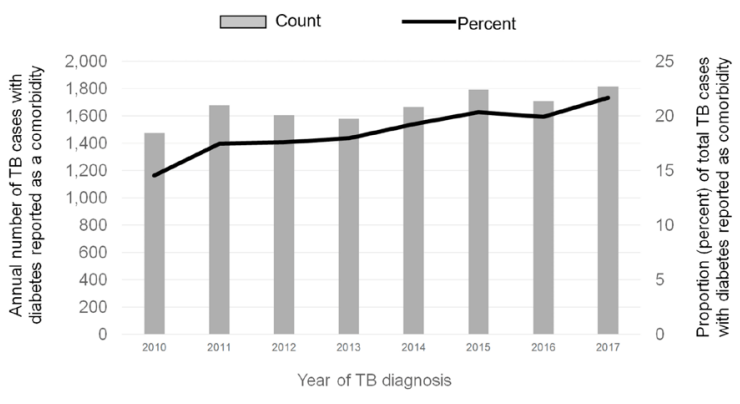

Figure 1 Diabetes reported as a comorbidity among adults with newly diagnosed tuberculosis (TB) disease, as annual count and proportion of total TB cases, USA, 2010-2017.

examine TB disease characteristics and patient outcomes associated with diabetes.

\section{METHODS}

\section{Data source and definitions}

We analyzed all verified TB cases reported to NTSS from the 50 US states and District of Columbia for 1 January 2010 to 31 December 2017. We excluded 6535 patients with TB aged $<20$ years because only 45 of these young people were also reported as having diabetes. We also excluded 13 persons with unknown age, none of whom were reported as having diabetes. During 2010-2017, a total of 71855 persons aged $\geq 20$ years were reported as having TB disease. Standard NTSS patient-level variables include demographics, clinical characteristics, and social risk factors associated with TB (eg, homelessness and substance use). Nativity was considered US-born if the patient had been born in the USA, a US territory, or abroad to at least one US citizen parent.

The diabetes variable, added in 2009, has been reported by all reporting areas since 2010. NTSS defines diabetes as diagnosis of diabetes mellitus either before or concurrent with the TB diagnosis. ${ }^{9}$ Health department staff completing the NTSS form must verify that the diagnosis

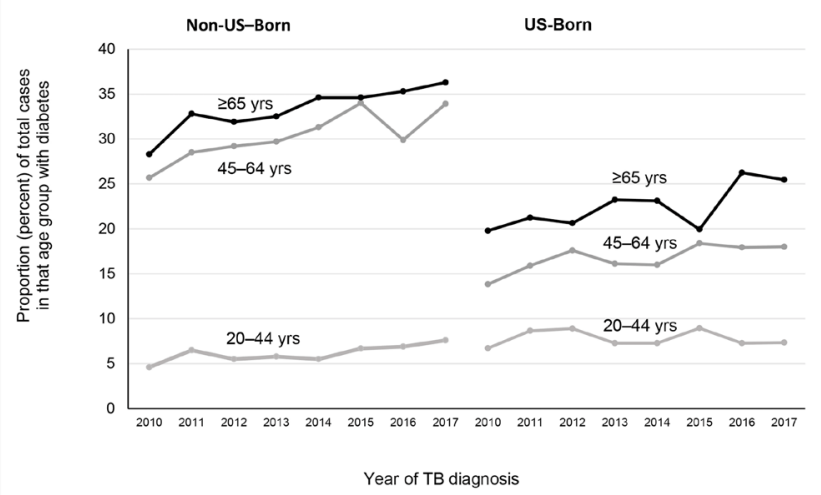

Figure 2 Age-specific prevalence of diabetes reported as a comorbidity among adults with tuberculosis (TB), by nativity and year, USA, 2010-2017. is documented by a healthcare provider, but whether it is type 1 or 2 is not collected. Further, until 2020, NTSS did not collect any diabetes-related laboratory results, nor did it have a variable to indicate that diabetes had been ruled out (ie, anyone without a known diabetes diagnosis was coded for this analysis as having unknown diabetes status). Additional medical comorbidities collected in NTSS include HIV infection, end-stage renal disease, history of organ transplantation, or immunosuppression caused by another medical problem.

\section{Prevalence calculations}

For each year during 2010-2017, we determined the annual number and proportion of adults with incident $\mathrm{TB}$ who were reported as also having diabetes. To more accurately compare diabetes prevalence across racial/ ethnic groups of patients with TB, age-adjusted prevalence for each racial/ethnic group was calculated by using the 2010 US population standard. ${ }^{10}$

\section{Statistical analysis}

We used logistic regression to assess which characteristics were associated with a reported diabetes diagnosis. Our multivariable model initially included any variables that were significant $(p<0.05)$ on univariate analysis. The referent group was persons with unknown diabetes status, and we separately analyzed those aged $20-44$ years and those aged $\geq 45$ years. $P$ values for comparing risk factors with continuous counts (eg, time to sputum conversion and time to treatment completion) were obtained by using the Wilcoxon rank-sum test.

\section{Patient protections}

NTSS operates under an Assurance of Confidentiality issued under Sections 306 and 308(d) of the US Public Health Service Act (42 USC 242k and 242m(d)). TB case reports were collected as part of routine public health surveillance; this retrospective analysis using existing and non-identifiable data was not considered research and did not require approval by an institutional review board.

\section{RESULTS}

\section{Prevalence of diabetes among patients with TB}

Of the 71855 persons aged $\geq 20$ years who were reported as having TB disease during 2010-2017, 18\% were reported as also having a diagnosis of diabetes $(\mathrm{n}=13281)$. Across the 8-year period, the annual proportion of adults with TB who also had diabetes increased from $15 \%$ in 2010 to $22 \%$ in 2017 , and the annual case count of adults with both conditions increased from 1469 to 1815 (figure 1).

\section{Descriptive results}

Non-US-born patients with TB aged $\geq 45$ years had the highest prevalence of diabetes (figure 2). After age adjustment, Native Hawaiian/Other Pacific Islander patients with TB had the highest prevalence of diabetes (37\%), followed by Hispanic patients (23\%) and non-Hispanic Asians (17\%). (Age-adjusted prevalence by race/ethnicity 
Table 1 Characteristics of adults with tuberculosis, by diabetes status, USA, 2010-2017

\begin{tabular}{|c|c|c|c|c|c|}
\hline \multirow[b]{2}{*}{ Characteristic } & \multicolumn{2}{|c|}{$\begin{array}{c}\text { Diagnosed diabetes } \\
n=13281\end{array}$} & \multicolumn{2}{|c|}{$\begin{array}{l}\text { Diabetes status unknown } \\
\qquad \mathrm{n}=58574\end{array}$} & \multirow{2}{*}{$\begin{array}{l}\chi^{2} \\
\mathbf{P} \text { value }\end{array}$} \\
\hline & $\mathbf{n}$ & $\%$ & $\mathbf{n}$ & $\%$ & \\
\hline End-stage renal disease & 950 & 7.2 & 996 & 1.7 & $<0.001$ \\
\hline HIV infection & 258 & 1.9 & 4061 & 6.9 & $<0.001$ \\
\hline History of solid organ transplantation & 145 & 1.1 & 249 & 0.4 & $<0.001$ \\
\hline Immunosuppression caused by other medical problem & 773 & 5.8 & 3024 & 5.2 & 0.001 \\
\hline Ages 20-44 years & 1947 & 14.7 & 27670 & 47.2 & $<0.001$ \\
\hline Ages $45-64$ years & 6045 & 45.5 & 18307 & 31.3 & $<0.001$ \\
\hline Age $\geq 65$ years & 5289 & 39.8 & 12597 & 21.5 & $<0.001$ \\
\hline \multicolumn{6}{|l|}{ Self-reported race/ethnicity } \\
\hline Hispanic & 4465 & 33.6 & 15260 & 26.0 & $<0.001$ \\
\hline Non-Hispanic Asian & 5066 & 38.1 & 18729 & 32.0 & $<0.001$ \\
\hline Non-Hispanic black & 1942 & 14.6 & 13705 & 23.4 & $<0.001$ \\
\hline Non-Hispanic white & 1374 & 10.3 & 9333 & 15.9 & $<0.001$ \\
\hline American Indian/Alaska Native & 168 & 1.3 & 721 & 1.2 & 0.374 \\
\hline Native Hawaiian/Other Pacific Islander & 170 & 1.3 & 344 & 0.6 & $<0.001$ \\
\hline Other (unknown, missing, or multiple race) & 96 & 0.7 & 482 & 0.8 & 0.122 \\
\hline Non-US-born & 9734 & 73.3 & 38989 & 66.6 & $<0.001$ \\
\hline Female & 4690 & 35.3 & 22796 & 38.9 & $<0.001$ \\
\hline Known contact of person with infectious TB & 188 & 1.4 & 1615 & 2.8 & $<0.001$ \\
\hline Previous diagnosis of TB disease & 615 & 4.6 & 3073 & 5.2 & 0.001 \\
\hline Previous incomplete treatment of latent TB infection & 269 & 2.0 & 1758 & 3.0 & $<0.001$ \\
\hline \multicolumn{6}{|l|}{ Anatomic site of TB disease } \\
\hline Pulmonary only & 10172 & 76.6 & 39520 & 67.5 & $<0.001$ \\
\hline Both pulmonary and extrapulmonary & 1158 & 8.7 & 6095 & 10.4 & $<0.001$ \\
\hline Extrapulmonary only & 1947 & 14.7 & 12913 & 22.0 & $<0.001$ \\
\hline Sputum smear positive for acid-fast bacilli* & 6593 & 58.2 & 21089 & 46.2 & $<0.001$ \\
\hline Lung cavity visible on chest radiograph* & 3717 & 32.8 & 11032 & 24.2 & $<0.001$ \\
\hline TB resistant to isoniazid $\dagger$ & 1050 & $9.5^{\star}$ & 4136 & $9.2^{*}$ & 0.275 \\
\hline TB resistant to rifampin $\dagger$ & 167 & $1.5^{\star}$ & 765 & $1.7^{\star}$ & 0.088 \\
\hline $\begin{array}{l}\text { Multidrug-resistant TB (resistant to at least isoniazid } \\
\text { and rifampin) } \dagger\end{array}$ & 139 & $1.3^{*}$ & 637 & $1.4^{*}$ & 0.069 \\
\hline Homeless within the year before TB diagnosis & 514 & 3.9 & 3500 & 6.0 & $<0.001$ \\
\hline Excess alcohol use within the year before TB diagnosis & 1189 & 9.0 & 7091 & 12.1 & $<0.001$ \\
\hline Injection drug use within the year before TB diagnosis & 143 & 1.1 & 940 & 1.6 & $<0.001$ \\
\hline $\begin{array}{l}\text { Non-injection drug use within the year before TB } \\
\text { diagnosis }\end{array}$ & 546 & 4.1 & 4636 & 7.9 & $<0.001$ \\
\hline $\begin{array}{l}\text { Resident of a correctional facility at time of TB } \\
\text { diagnosis }\end{array}$ & 165 & 1.2 & 2712 & 4.6 & $<0.001$ \\
\hline $\begin{array}{l}\text { Resident of a long-term care facility at time of TB } \\
\text { diagnosis }\end{array}$ & 443 & 3.3 & 1106 & 1.9 & $<0.001$ \\
\hline
\end{tabular}

*Denominators for these proportions are based on the 11330 adults with diagnosed diabetes and the 45615 adults with unknown diabetes status who had a pulmonary site of TB disease.

$\dagger$ †enominators for these proportions are based on the 11059 adults with diagnosed diabetes and the 44749 adults with unknown diabetes status whose Mycobacterium tuberculosis culture also underwent drug-susceptibility testing.

TB, tuberculosis.

is not shown in table 1 , where the presented proportions are based on diabetes status.)

Approximately half of patients with TB who also had diabetes had pulmonary forms of TB that were smear positive for acid-fast bacilli, and nearly a third had evidence of cavitary TB on chest radiograph (table 1 ). Social risk factors for $\mathrm{TB}$, including incarceration at the time of diagnosis, recent homelessness, and recent substance misuse, were less prevalent among patients with TB and diabetes. An exception to this pattern was residence in a 

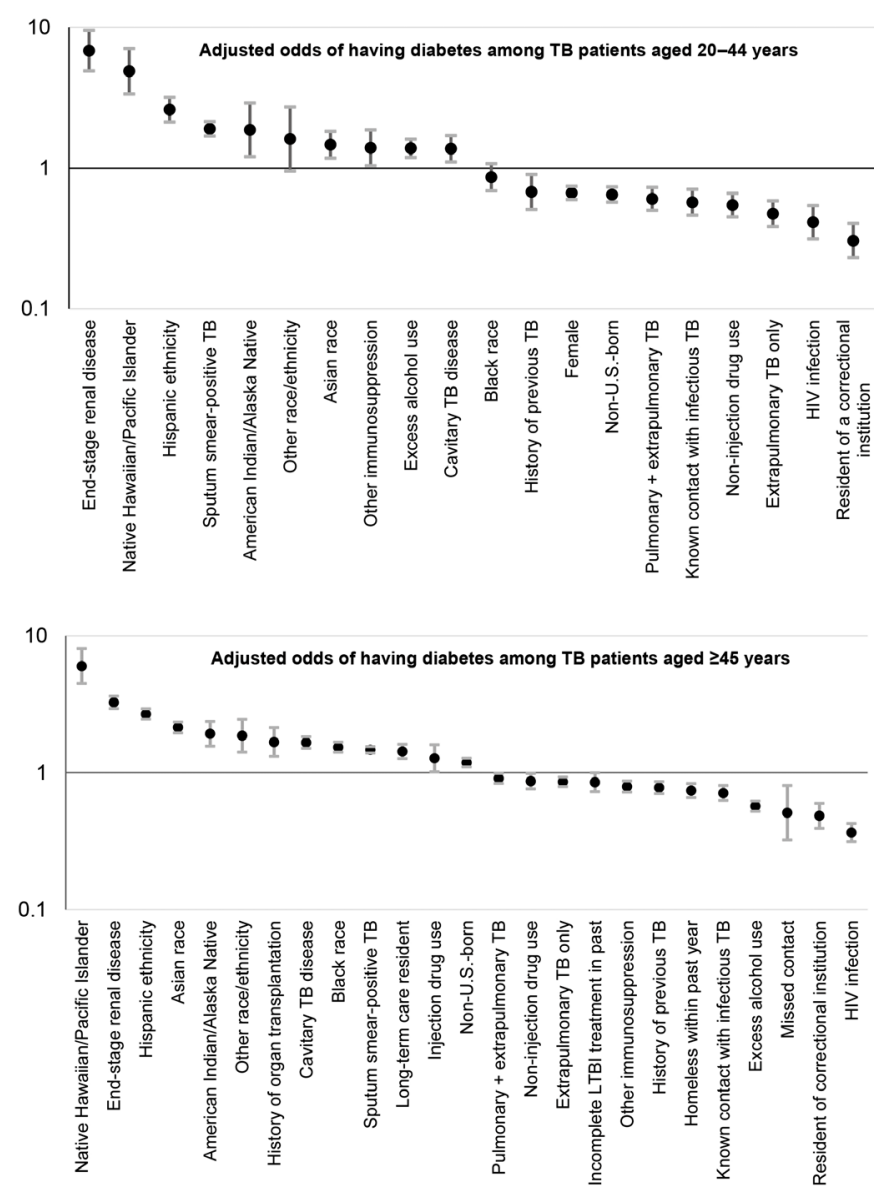

Figure 3 Adjusted odds of having diabetes reported as a comorbidity among adults with tuberculosis (TB), by age group, USA, 2010-2017. LTBI, latent TB infection.

long-term care facility at diagnosis, which was more prevalent among patients with $\mathrm{TB}$ who also had diabetes as a comorbidity (table 1 ).

\section{Multivariable model results}

Figure 3 presents the adjusted odds of having diabetes as a comorbidity at the time of TB diagnosis; the ORs are presented in descending order from the strongest positive association to the strongest negative association. All variables included in the two age-based models are shown in figure 3. The adjusted odds of also having diabetes was higher among patients with end-stage renal disease (adjusted OR (aOR) if aged 20-44 years: 6.8; 95\% CI 4.9 to 9.5 ; aOR if aged $\geq 45$ years: 3.3 ; $95 \%$ CI 2.9 to 3.6 ). Among patients with TB aged 20-44 years, lower odds of diabetes was associated with residence in a correctional facility (aOR 0.3 ; 95\% CI 0.2 to 0.4 ), HIV infection (aOR $0.4 ; 95 \%$ CI 0.3 to 0.5 ), and non-US-born nativity (aOR $0.7 ; 95 \%$ CI 0.6 to 0.7 ). In contrast, non-US-born nativity was positively associated (aOR 1.2; 95\% CI 1.1 to 1.3 ) with diabetes among older patients with TB. Among patients with TB aged 20-44 years with diabetes, the aOR for cavitary or sputum smear-positive TB was 1.4 (95\% CI 1.1 to 1.7) and 1.9 (95\% CI 1.7 to 2.1), respectively, compared with that of patients in the same age group without a diabetes diagnosis reported. Among patients with TB aged $\geq 45$ years with diabetes, the aOR for cavitary or sputum smear-positive TB was 1.7 (95\% CI 1.5 to 1.8 ) and 1.5 (95\% CI 1.4 to 1.6), respectively, compared with that of patients in the same age group without a diabetes diagnosis reported.

\section{Patient outcomes}

Patients with TB who had diabetes as a known comorbidity were more likely to die, either before TB treatment could begin or during treatment (table 2). However, the deceased patients with diabetes had an older age distribution than those with unknown diabetes status (table 2). Even after accounting for this older age distribution and excluding the deaths at time of diagnosis, patients with TB and diabetes had 1.3 (95\% CI 1.1 to 1.5) the adjusted odds of death during the monthslong treatment period for TB.

After excluding all deaths, TB treatment completion was $>93 \%$ for both adults with and without diabetes reported (table 2). However, those with diabetes required slightly more time to attain negative $M$. tuberculosis cultures (median days to sputum conversion: 47 vs $42 ; \mathrm{p}<0.001)$ and an additional month to complete treatment (median days to complete TB treatment: 249 vs 212; $\mathrm{p}<0.001)$.

\section{DISCUSSION}

Even as overall TB case counts declined $18 \%$ in the USA during $2010-2017,{ }^{8}$ the prevalence of diabetes as a reported comorbidity among adults with $\mathrm{TB}$ disease steadily increased from $15 \%$ in 2010 to $22 \%$ in 2017 . An initial interpretation might be that these findings are not surprising - given the high prevalence of diagnosed and undiagnosed diabetes in the USA-but the age-adjusted prevalence of diabetes during the same period is thought to have stabilized at an estimated $9.4 \%$ of the adult US population. ${ }^{11}$

An important limitation of this analysis is potential misclassification (under-reporting) of diabetes as a comorbidity. The 58574 patients listed in table 1 as 'diabetes status unknown' include both adults who had diabetes ruled out and adults who were not evaluated for diabetes. Although this analysis could not discriminate between the two possibilities, that important distinction is part of the NTSS case report form revised in 2020. Diabetes was added to the form in 2009, so one might expect that health departments more systematically ascertained diabetes status toward the end of the 20102017 period. In addition, screening for diabetes might have increased after the 2016 recommendation to screen all patients aged $\geq 45$ years at the start of TB treatment. ${ }^{2}$ Nevertheless, a strength of this first national analysis of diabetes among persons newly diagnosed with $\mathrm{TB}$ disease is that it is based on 71855 patients with $\mathrm{TB}$, including 13281 reported to have diabetes as a comorbidity. 
Table 2 Outcomes among adults with tuberculosis, by diabetes status, USA, 2010-2015*

\begin{tabular}{|c|c|c|c|c|c|}
\hline \multirow[b]{2}{*}{ Patient outcome } & \multicolumn{2}{|c|}{$\begin{array}{l}\text { Diagnosed diabetes } \\
n=9762\end{array}$} & \multicolumn{2}{|c|}{$\begin{array}{l}\text { Diabetes status unknown } \\
\qquad n=45162\end{array}$} & \multirow{2}{*}{$\begin{array}{l}\chi^{2} \\
\mathrm{P} \text { value }\end{array}$} \\
\hline & $\mathbf{n}$ & $\%$ & $\mathbf{n}$ & $\%$ & \\
\hline (Cause of death not documented for these patients) & - & - & - & - & - \\
\hline Ages 20-44 years & 9 & 0.1 & 160 & 14.8 & \\
\hline Began TB treatment but died before treatment completion & 1052 & 10.8 & 2610 & 5.8 & $<0.001$ \\
\hline TB considered a cause of death $\dagger$ & 361 & 34.3 & 977 & 37.4 & 0.038 \\
\hline Ages $20-44$ years & 44 & 4.2 & 277 & 10.6 & \\
\hline $45-64$ years & 301 & 28.6 & 826 & 31.6 & \\
\hline Refused to complete TB treatment & 64 & 0.7 & 349 & 0.8 & 0.112 \\
\hline Reason for not completing treatment not documented & 76 & 0.8 & 371 & 0.8 & 0.334 \\
\hline $\begin{array}{l}\text { Completed TB treatment, with all patients with TB as } \\
\text { denominator }\end{array}$ & 7867 & 80.6 & 38624 & 85.5 & $<0.001$ \\
\hline $\begin{array}{l}\text { Completed TB treatment, excluding deaths from } \\
\text { denominator }\end{array}$ & 7867 & 93.6 & 38624 & 93.1 & 0.063 \\
\hline
\end{tabular}

*Because of the monthslong duration of TB treatment, outcome data can require up to 2 years after the initial case report; therefore, patients with incident TB disease in 2016 and 2017 were excluded from this table. Had we included them, the proportions of patients who died before TB treatment could be started were similar: 375 (2.8\%) among the 13281 adult patients with TB with diagnosed diabetes and $1303(2.2 \%)$ among the 58574 with unknown diabetes status during 2010-2017.

†Denominators for these proportions are based on the 1052 adult patients with TB with diagnosed diabetes and the 2610 with unknown diabetes status who began TB treatment but died before achieving treatment completion.

$\ddagger$ Denominators for these proportions are based on the 8408 adults with diagnosed diabetes and the 41471 adults with unknown diabetes status who began TB treatment and did not die during treatment.

TB, tuberculosis.

Despite this potential misclassification (which would be predicted to bias comparisons toward the null), having diabetes as a comorbidity at time of TB diagnosis appears associated with more severe presentations and poorer outcomes in the USA. Patients with TB who were aged $\geq 45$ years and had diabetes had $70 \%$ greater odds of having cavitary TB and $50 \%$ greater odds of smear-positive TB. We also found $30 \%$ greater odds of dying during treatment. These findings are consistent with previous studies from other parts of the world. ${ }^{3-6} 12$

In our US analysis, patients with diabetes responded more slowly to TB treatment, as evidenced by a median of 5 more days to attain negative $M$. tuberculosis cultures. TB treatment regimens for persons with diabetes took a median of 37 days longer to complete. But in contrast with the findings of other studies, ${ }^{46}$ having diabetes did not appear to impede TB treatment completion, despite more complicated case management features, including end-stage renal disease, postorgan transplantation, and long-term care settings. In fact, after excluding deaths, TB treatment completion was $>93 \%$ (table 2). And although a 2011-2014 study in Tbilisi, Georgia, found that patients with diabetes were more likely to have drugresistant $\mathrm{TB},{ }^{12}$ our study found that patients with both TB disease and diabetes in the USA during 2010-2017 were not (table 1).

Our findings underscore the recent national recommendation for baseline diabetes screening among all patients with TB aged $\geq 45$ years and all younger patients with risk factors for diabetes (ie, body mass index $>25 \mathrm{~kg}$ / $\mathrm{m}^{2}$; first-degree relative with diabetes; Hispanic ethnicity; or African-American, Asian, American Indian/Alaska Native, or Hawaiian Native/Pacific Islander race).$^{2}$ Like other infections, M. tuberculosisinfection can worsen blood glucose control and complicate the clinical management of diabetes. Further, Harries et al have appealed for TB screening among persons with diabetes (ie, bidirectional screening). ${ }^{13}$ Indeed, global concern about the 'converging epidemics of tuberculosis and diabetes' has helped draw attention to the synergistic influence each might have on the other. ${ }^{1415}$

The progression of untreated latent $\mathrm{TB}$ infection (LTBI) causes most of the TB disease in the USA. ${ }^{8}$ In older persons, diabetes and the ageing process itself synergistically reduce levels of interferon gamma, impairing cellmediated immunity and helping to activate progression of long-standing LTBI. ${ }^{16}$ Both TB disease and LTBI in the USA occur primarily among non-US-born persons. ${ }^{817} 18$ 
Preventing active, infectious forms of TB disease thus requires scaling up of interventions to detect and treat LTBI in this population. ${ }^{19}$

The International Diabetes Federation projects that 693 million adults will have diabetes by $2024 .{ }^{20}$ Our analysis revealed that non-US-born patients with TB aged $\geq 45$ years were reported as having a much higher prevalence of diabetes than US-born patients with TB in the same age group (figure 2), suggesting that the increasing diabetes prevalence worldwide could be affecting TB's epidemiology in the USA. Addressing that challenge will require coordination between the private sector, where case management of diabetes often resides, and the public health sector, which has been charged with the responsibility for controlling and eventually eliminating TB in the USA.

Acknowledgements We gratefully acknowledge the contributions and efforts of local and state health departments that report cases into the National Tuberculosis Surveillance System. We thank Kristen Renneker for her earlier unpublished analysis with 2009-2011 data and C Kay Smith for editorial services.

Contributors LRA conceived and executed the analysis. JSK provided analytic assistance. JSK and MBH reviewed interim results, provided interpretation, and suggested additional analyses. LRA created the first draft of the article, and MBH revised the article. All authors approved the final version.

Funding This is a publicly funded research by employees of the Centers for Disease Control and Prevention, Atlanta, Georgia.

Disclaimer The findings and conclusions in this report are those of the authors and do not necessarily represent the official position of the Centers for Disease Control and Prevention.

\section{Competing interests None declared.}

Patient consent for publication Not required.

Provenance and peer review Not commissioned; externally peer reviewed.

Data availability statement The National Tuberculosis Surveillance System operates under an Assurance of Confidentiality issued by the Centers for Disease Control and Prevention (CDC) under Sections 306 and 308(d) of the Public Health Service Act (42 USC 242k and 242m(d)). Data are reported voluntarily to $\mathrm{CDC}$ by state and local health departments on a case report form called the Report of Verified Case of Tuberculosis (OMB No. 0920-0026). The Assurance of Confidentiality prevents disclosure of any information that could be used to directly or indirectly identify patients. For more information, see the CDC/ATSDR Policy on Releasing and Sharing Data (at http://www.cdc.gov/maso/Policy/ReleasingData. pdf). A limited dataset is available at http://wonder.cdc.gov/TB-v2013.html. Researchers may apply to analyze additional data at CDC headquarters by contacting TBInfo@cdc.gov.

Open access This is an open access article distributed in accordance with the Creative Commons Attribution Non Commercial (CC BY-NC 4.0) license, which permits others to distribute, remix, adapt, build upon this work non-commercially, and license their derivative works on different terms, provided the original work is properly cited, appropriate credit is given, any changes made indicated, and the use is non-commercial. See: http://creativecommons.org/licenses/by-nc/4.0/.

ORCID ID

Maryam B Haddad http://orcid.org/0000-0001-6327-068X
REFERENCES

1 Lönnroth K, Roglic G, Harries AD. Improving tuberculosis prevention and care through addressing the global diabetes epidemic: from evidence to policy and practice. Lancet Diabetes Endocrinol 2014;2:730-9.

2 Nahid P, Dorman SE, Alipanah N, et al. Official American Thoracic Society/Centers for Disease Control and Prevention/Infectious Diseases Society of America clinical practice guidelines: treatment of drug-susceptible tuberculosis. Clin Infect Dis 2016;63:e147-95.

3 Zafar MI, Chen L-L, Xiaofeng Y, et al. Impact of diabetes mellitus on radiological presentation of pulmonary tuberculosis in otherwise non-immunocompromised patients: a systematic review. Curr Med Imaging Rev 2019;15:543-54.

4 Jiménez-Corona ME, Cruz-Hervert LP, García-García L, et al. Association of diabetes and tuberculosis: impact on treatment and post-treatment outcomes. Thorax 2013;68:214-20.

$5 \mathrm{Ma} \mathrm{Y}$, Huang ML, Li T, et al. Role of diabetes mellitus on treatment effects in drug-susceptible initial pulmonary tuberculosis patients in China. Biomed Environ Sci 2017;30:671-5.

6 Baker MA, Harries AD, Jeon CY, et al. The impact of diabetes on tuberculosis treatment outcomes: a systematic review. BMC Med 2011;9:81.

7 Nguyen $\mathrm{CH}$, Pascopella L, Barry PM. Association between diabetes mellitus and mortality among patients with tuberculosis in California, 2010-2014. Int J Tuberc Lung Dis 2018;22:1269-76.

8 Armstrong LR, Winston CA, Stewart B, et al. Changes in tuberculosis epidemiology, United States, 1993-2017. Int J Tuberc Lung Dis 2019;23:797-804.

9 Centers for Disease Control and Prevention (CDC). CDC tuberculosis surveillance data training - report of verified case of tuberculosis. Atlanta, GA: US Department of Health and Human Services, CDC, 2009. https://www.cdc.gov/tb/programs/rvct/default.htm

10 Li C, Ford ES, Zhao G, et al. Age adjustment of diabetes prevalence: use of 2010 U.S. Census data. J Diabetes 2014;6:451-61.

11 Centers for Disease Control and Prevention (CDC). Diabetes report card 2017. Atlanta, GA: US Department of Health and Human Services, CDC, 2018. https://www.cdc.gov/diabetes/library/reports/ reportcard.html

12 Magee MJ, Kempker RR, Kipiani M, et al. Diabetes mellitus is associated with cavities, smear grade, and multidrug-resistant tuberculosis in Georgia. Int J Tuberc Lung Dis 2015;19:685-92.

13 Harries AD, Kumar AMV, Satyanarayana S, et al. Communicable and non-communicable diseases: connections, synergies and benefits of integrating care. Public Health Action 2015;5:156-7.

14 Magee MJ, Salindri AD, Gujral UP, et al. Convergence of noncommunicable diseases and tuberculosis: a two-way street? Int $J$ Tuberc Lung Dis 2018;22:1258-68.

15 Ronacher K, van Crevel R, Critchley JA, et al. Defining a research agenda to address the converging epidemics of tuberculosis and diabetes: Part 2: underlying biologic mechanisms. Chest 2017;152:174-80.

16 Menon S, Rossi R, Nshimyumukiza L, et al. Convergence of a diabetes mellitus, protein energy malnutrition, and TB epidemic: the neglected elderly population. BMC Infect Dis 2016;16:361.

17 Barron MM, Shaw KM, Bullard KM, et al. Diabetes is associated with increased prevalence of latent tuberculosis infection: findings from the National Health and Nutrition Examination Survey, 2011-2012. Diabetes Res Clin Pract 2018;139:366-79.

18 Miramontes R, Hill AN, Yelk Woodruff RS, et al. Tuberculosis infection in the United States: prevalence estimates from the National Health and Nutrition Examination Survey, 2011-2012. PLoS One 2015;10:e0140881.

19 US Preventive Services Task Force, Bibbins-Domingo K, Grossman $\mathrm{DC}$, et al. Screening for latent tuberculosis infection in adults: US Preventive Services Task Force recommendation statement. JAMA 2016;316:962-9.

20 Cho NH, Shaw JE, Karuranga S, et al. IDF diabetes atlas: global estimates of diabetes prevalence for 2017 and projections for 2045. Diabetes Res Clin Pract 2018;138:271-81. 